\title{
Intrarater and Interrater Reliability of a Passive Shoulder Flexion Range of Motion Measurement for Latissimus Dorsi Flexibility in Young Competitive Swimmers
}

\author{
Stef Feijen, Angela Tate, Kevin Kuppens, Thomas Struyf, Anke Claes, and Filip Struyf
}

\begin{abstract}
Context: The latissimus dorsi plays a major role in generating the propulsive force during swimming. In addition, stiffness of this muscle may result in altered stroke biomechanics and predispose swimmers to shoulder pain. Measuring the flexibility of the latissimus dorsi can be of interest to reduce injury. However, the reliability of such measurement has not yet been investigated in competitive swimmers. Objective: To assess the within-session intrarater and interrater reliability of a passive shoulder flexion range of motion measurement for latissimus dorsi flexibility in competitive swimmers. Design: Within-session intrarater and interrater reliability. Setting: Competitive swimming clubs in Flanders, Belgium. Participants: Twenty-six competitive swimmers (15.46 [2.98] y; 16 men and 10 women). Intervention: Each rater performed 2 alternating (eg, left-right-left-right) measurements of passive shoulder flexion range of motion twice, with a 30-second rest period in between. Main Outcome Measures: The intraclass correlation coefficients were calculated to assess intrarater and interrater reliability. Results: Interrater intraclass correlation coefficient ranged from .54 (95\% confidence interval [CI], -.16 to .81) to .57 (95\% CI, -.24 to .85 ). Results for the intrarater reliability ranged from .91 (95\% CI, .81 to .96) to .94 (95\% CI, .87 to .97). Conclusion: Results of this study suggest that shoulder flexion range of motion in young competitive swimmers can be measured reliably by a single rater within the same session.
\end{abstract}

Keywords: swimming, articular, outcome assessment

The latissimus dorsi (LD) primarily induces extension, adduction, and internal rotation of the arm ${ }^{1}$ and, hereby, greatly contributes to the forward propulsive power during freestyle swimming. ${ }^{2}$ Prior to delivering this propulsive force, the swimmer typically strives for a maximum available reach, but this requires sufficient shoulder flexion range of motion (ROM). ${ }^{3,4}$ Adequate flexibility of the LD muscle also allows the shoulder to reach full motion by enhancing both lateral rotation of the humerus and scapular upward rotation. ${ }^{4}$

Competitive swimmers are often exposed to tremendous training volumes, swimming up to $14,000 \mathrm{~m}$ each day. ${ }^{5}$ The constant loading of the LD that comes with this repetitive swim training will likely result in muscle hypertrophy but could also lead to increased muscular stiffness and resistance to elongation. ${ }^{6}$ However, stiffness of the LD can limit both scapular and glenohumeral $\mathrm{ROM}^{7}$ and, thereby, alter the swimmer's stroke biomechanics. Such adaptations have previously been associated with greater levels of shoulder pain and disability. ${ }^{8}$

The essential role of the LD in the swimming stroke highlights the importance of reliably quantifying its flexibility in competitive swimmers. A method often used in clinical setting is to assess passive shoulder flexion ROM as described by the LD flexibility (LDF) measurement. ${ }^{9}$ This method has been reported to show poor reproducibility between sessions in healthy adults. ${ }^{10}$ However, its reliability has not yet been investigated in competitive swimmers

Feijen, Kuppens, Claes, and F. Struyf are with the Department of Rehabilitation Sciences and Physiotherapy, Faculty of Medicine and Health Sciences, University of Antwerp, Antwerp, Belgium. Tate is with the Department of Physical Therapy, Arcadia University, Glenside, PA, USA. T. Struyf is with the Department of Public Health and Primary Care, KU Leuven, Leuven, Belgium. F. Struyf (Filip.struyf@ uantwerpen.be) is corresponding author. who may experience changes in physical characteristics of the muscle. Consequently, this study aims to investigate the withinsession intrarater and interrater reliability of measuring shoulder flexion ROM for LDF in competitive swimmers aged 10-20 years.

\section{Methods}

\section{Design}

Prior to the study, rater 1 (S.F., master in rehabilitation sciences and physiotherapy with $3 \mathrm{y}$ of clinical experience) was trained by an experienced physiotherapist (K.K., master in rehabilitation sciences and physiotherapy with over $10 \mathrm{y}$ of clinical experience) in performing accurate measurements of passive shoulder flexion ROM for LDF. ${ }^{9}$ Afterward, rater 2 (L. Wouters, bachelor in rehabilitation sciences and physiotherapy) received a similar training, which was then given by rater 1 .

Swimmers were tested during their swim-training session and familiarized with the movement before data collection. The order of testing (order of rater and primary side of testing) was randomized by block design. Both shoulders were measured independently using a fluid-filled bubble inclinometer (Plurimeter; Dr. Rippstein, Saint-Sulpice, Switzerland). Raters were blinded to each other's findings.

\section{Participants}

Swimmers were included if they trained for an average of at least 4 hours per week and were active in competitive swimming in Belgium. Exclusion criteria were swimmers younger than 10 years; having shoulder pain or trauma within the past 12 months; or the presence of any neurological, systemic, metabolic, rheumatologic, 
or cardiovascular disease. This study has been approved by the Committee for Medical Ethics UZA-UAntwerp (B300201630081). Subjects gave written informed consent prior to the work.

\section{Procedure}

First, swimmers completed a baseline questionnaire regarding demographics and sport-specific features. Next, passive shoulder flexion ROM was assessed twice on each side alternatively (eg, left-right-left-right). Following a 30-second rest period, this procedure was then repeated by the same rater using counterbalancing for primary side of testing (eg, right-left-right-left). At the end of the prospective screening session, the entire procedure was again counterbalanced and performed by the other rater.

Passive ROM of both shoulders was assessed with the subject lying supine, with the knees and hips flexed and the humerus externally rotated next to the body. The rater placed the inclinometer on the anterior aspect of the humerus, with the base of the instrument placed parallel to the length of the humerus (Figure 1A). Next, the rater assessed ROM passively by flexing the shoulder in the sagittal plane until the point of a firm muscular end-feeling. The participant was asked to maintain an active abdominal contraction during the entire movement and relax while maintaining it in external rotation and extension. Finally, the rater noted the degree indicated by the inclinometer's pointer (Figure 1B).

Standardized oral instructions for positioning of the participant, active contraction, and relaxation were given during the procedure. Criteria that resulted in a failed test and repeating the movement were loss of upper-extremity position (humeral external rotation and elbow extension), loss of posterior pelvic tilt (inability to maintain abdominal muscle contraction during movement), or loss of lower-extremity position (inability to maintain hip and knee flexion or keep the feet flat on the table).
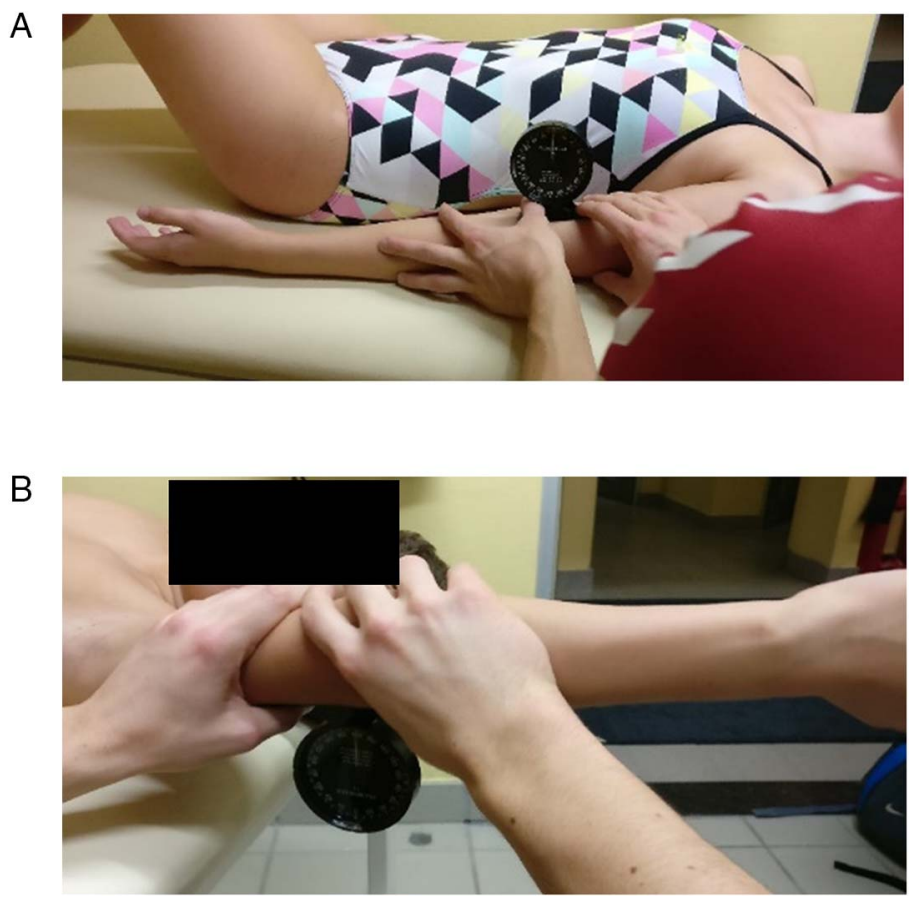

Figure 1 - (A) Begin position of the passive shoulder flexion range of motion measurement and (B) ending position of the passive shoulder flexion range of motion measurement.

\section{Statistical Analysis}

The required sample size was determined using estimates for correlation, $r(\mathrm{~N}=22$, effect size $=.50$, power $=.80)$ and 2 -way analysis of variance, $F(\mathrm{~N}=17$, effect size $=.50$, power $=.80, \mathrm{df}=$ 1). ${ }^{10,11}$ All variables were tested for normality using the ShapiroWilk test $(P>.05)$. Intraclass correlation coefficient (ICC) model 2,4 (2-way random-effects model-absolute agreement) and model 3,2 (2-way mixed-effects model-absolute agreement) were used to analyze the interrater and intrarater reliability, respectively. Interrater reliability was calculated using the mean values of all 4 trials per shoulder. For intrarater reliability, the average values of the first and second 2 trials were compared for each shoulder separately. To calculate the measurement error associated with the LDF measurement, both SEM $(\mathrm{SEM}=\mathrm{SD} \times \sqrt{ }(1-\mathrm{ICC}))$ and minimal detectable change at the $95 \%$ confidence level $\left(\mathrm{MDC}_{95}=\right.$ $\mathrm{SEM} \times 1.96 \times \sqrt{ } 2$ ) were used. ${ }^{12}$ Reliability coefficients were interpreted as follows: $<.50$, poor; .50 to .75 , moderate; .76 to .90 , good; and $>.90$, excellent. ${ }^{11}$

\section{Results}

Demographic characteristics of the swimmers $(\mathrm{N}=26)$ are presented in Table 1 . Table 2 represents the intrarater and interrater ICC values as well as their $95 \%$ confidence interval (CI). Reliability analyses were conducted separately for the left and right side.

Within-session interrater reliability analysis showed ICC values of $.54(95 \% \mathrm{CI},-.16$ to .81$)$ and .57 (95\% CI, -.24 to .85$)$ for the right and left shoulder flexion measurement, respectively. Intrarater reliability within rater 1 ranged from .93 (95\% CI, .84 to $.97)$ to .94 (95\% CI, .87 to .97). Rater 2 showed intrarater ICCs of $.91(95 \%$ CI, .81 to .96) for the left and .92 (95\% CI, .82 to .97) for the right shoulder flexion measurement.

\section{Discussion}

To our knowledge, this is the first study investigating the reliability of measuring shoulder flexion ROM for LDF in competitive swimmers. ICCs indicated that this test can be used reliably by a single rater within the same session $(\mathrm{ICC}=.91-.94)$. Interrater analyses, however, suggest very low reliability between raters as the CIs provide no evidence that the true ICC values are different from zero. Current SEMs ranged from $2.70^{\circ}$ to $3.59^{\circ}$ for intrarater reliability and from $6.48^{\circ}$ to $6.89^{\circ}$ for interrater reliability. These values fall in line with previously reported measurement errors of shoulder flexion ROM. ${ }^{4,10}$ SEMs indicate the precision of a single measurement but can also be used to calculate $\mathrm{MDC}_{95}$. MDCs ranged from $7.49^{\circ}$ to $9.94^{\circ}$ for intrarater reliability and from $17.95^{\circ}$ to $19.10^{\circ}$ for interrater reliability. These values reflect the amount of change that must be achieved in the measurement to be $95 \%$ sure that this change is determined by "true" individual changes beyond measurement error.

Current results are in line with previous research, reporting excellent intrarater reliability of shoulder flexion ROM in healthy controls within a single session. ${ }^{4}$ However, compared with the authors' data, previously performed studies ${ }^{4,10}$ consistently reported lower values of shoulder flexion ROM. These differences may mainly be explained by the characteristics of the study population. First, the participants included in this study were consistently younger, and while research suggests that for certain shoulder complex motions, children show greater ROM values than adults, ${ }^{13}$ this may partially explain the higher values obtained in this study. 
Table 1 Participant Demographics (Mean [SD] or the Frequency)

\begin{tabular}{lccc}
\hline Characteristics & Women $(\mathbf{n}=\mathbf{1 0})$ & Men $(\mathbf{n}=\mathbf{1 6})$ & Total $(\mathbf{N}=\mathbf{2 6})$ \\
\hline Age, y & $13.83(2.45)$ & $16.48(2.89)$ & $15.46(2.98)$ \\
Years in competition, y & $3.83(1.86)$ & $5.70(2.1)$ & $4.95(2.18)$ \\
Height, cm & $156.45(13.94)$ & $173.16(13.71)$ & $166.73(15.86)$ \\
Weight, kg & $48.3(14.58)$ & $61(12.93)$ & $56.12(14.71)$ \\
Arm span, cm & $159.3(15.48)$ & $175.44(14.13)$ & $169.23(16.44)$ \\
Weekly swim sessions & $4.50(0.71)$ & $5(1.25)$ & $4.80(1.08)$ \\
Training volume, h/wk & $8.50(1.68)$ & $9.60(2.99)$ & $9.16(2.56)$ \\
Right-hand dominance & $10(100 \%)$ & $16(100 \%)$ & $26(100 \%)$ \\
\hline
\end{tabular}

Table 2 Within-Session Intrarater (ICC 3,2) and Interrater Reliability (ICC 2,4)

\begin{tabular}{|c|c|c|c|c|c|c|}
\hline & \multicolumn{3}{|c|}{ Right side } & \multicolumn{3}{|c|}{ Left side } \\
\hline & ICC (95\% Cl) & SEM, deg & $\mathrm{MDC}_{95}$, deg & ICC (95\% CI) & SEM, deg & $\mathrm{MDC}_{95}, \mathrm{deg}$ \\
\hline \multicolumn{7}{|l|}{ Intrarater } \\
\hline Rater 1 & .93 (.84 to .97$)$ & 2.70 & 7.49 & .94 (.87 to .97$)$ & 2.72 & 7.54 \\
\hline Rater 2 & $.92(.82$ to .97$)$ & 3.26 & 9.03 & $.91(.81$ to .96$)$ & 3.59 & 9.94 \\
\hline \multicolumn{7}{|l|}{ Interrater } \\
\hline Raters 1-2 & $.54(-.16$ to .81$)$ & 6.48 & 17.95 & $.57(-.24$ to .85$)$ & 6.89 & 19.10 \\
\hline
\end{tabular}

Abbreviations: CI, confidence interval; ICC, intraclass correlation coefficient (average measure); $\mathrm{MDC}_{95}$, minimal detectable change with $95 \%$ CI.

Second, it has been shown that competitive swimmers are exposed to tremendous training volumes..$^{5}$ This repetitive nature of swimming and the large amount of shoulder revolutions that come with training may result in a task-specific increased ROM of the shoulder compared with healthy nonswimmers. ${ }^{14}$ Moreover, previous research investigating the $\mathrm{LDF}$ in competitive swimmers also reported greater values of shoulder flexion ROM, but only in those without shoulder pain. ${ }^{8,15}$ These results may indicate that competitive swimming indeed requires full scapular and glenohumeral motion in order to achieve maximal performance and decrease the risk of injury ${ }^{4}$ and that the increase in shoulder flexion ROM may protect swimmers from developing shoulder pain.

This study has some limitations that should be addressed. First, differences in skills, experience, personal characteristics, or rater's training procedure may have influenced interrater reliability within a single session. Next, swimmers were tested during their swimtraining session, and measurements may have been influenced by training-induced physical changes or fatigue of the swimmer. Finally, this study examined the within-session reliability in swimmers aged 10-20 years with no short-term history of injury. Therefore, findings of this study may not be generalized to people outside this population or age group.

This study investigated the within-session reliability of shoulder flexion ROM in young competitive swimmers. Results demonstrate that the shoulder flexion ROM can be assessed reliably by a single rater within the same session. Further research is necessary to investigate the intersession reliability of this measurement and should recruit a larger sample size, including participants of a greater age range that present both with and without symptoms.

\section{Acknowledgments}

The authors would like to thank L. Wouters for her assistance in performing the measurements. This research did not receive any specific grant from funding agencies, commercial, or not-profit sectors. The authors declare that they have no conflict of interest.

\section{References}

1. Bogduk N, Johnson G, Spalding D. The morphology and biomechanics of latissimus dorsi. Clin Biomech. 1998;13(6):377-385. doi:10. 1016/S0268-0033(98)00102-8

2. Pink M, Perry JF, Browne AF, Scovazzo Ml, Kerrigan J. The normal shoulder during freestyle swimming: an electromyographic and cinematographic analysis of twelve muscles. Am J Sports Med. 1991;19(6): 569-576. PubMed ID: 1781492 doi:10.1177/036354659101900603

3. Bak K, Fauno P. Clinical findings in competitive swimmers with shoulder pain. Am J Sports Med. 1997;25(2):254-260. PubMed ID: 9079184 doi: $10.1177 / 036354659702500221$

4. Herrington L, Horsley I. Effects of latissimus dorsi length on shoulder flexion in canoeists, swimmers, rugby players, and controls. J Sport Health Sci. 2014;3(1):60-63. doi:10.1016/j.jshs.2013.01.004

5. Sein ML, Walton J, Linklater J, et al. Shoulder pain in elite swimmers: primarily due to swim-volume-induced supraspinatus tendinopathy. Br J Sports Med. 2010;44(2):105-113. PubMed ID: 18463295 doi:10.1136/bjsm.2008.047282

6. Wilson GJ, Murphy AJ, Pryor JF. Musculotendinous stiffness: its relationship to eccentric, isometric, and concentric performance. $J$ Appl Physiol. 1994;76:2714-2719. PubMed ID: 7928905 doi:10. 1152/jappl.1994.76.6.2714

7. Laudner KG, Williams JG. The relationship between latissimus dorsi stiffness and altered scapular kinematics among asymptomatic collegiate swimmers. Phys Ther Sport. 2013;14(1):50-53. PubMed ID: 23312732 doi:10.1016/j.ptsp.2012.02.007

8. Tate A, Turner GN, Knab SE, Jorgensen C, Strittmatter A, Michener LA. Risk factors associated with shoulder pain and disability across the lifespan of competitive swimmers. J Athl Train. 2012;47(2):149158. doi:10.4085/1062-6050-47.2.149 
9. Kendall F, McCreary E, Provance P. Muscle Testing and Function With Posture and Pain. 4th ed. Philadelphia, PA: Lippincott Williams \& Wilkins; 1993.

10. Borstad JD, Briggs MS. Reproducibility of a measurement for latissimus dorsi muscle length. Physiother Theory Pract. 2010;26(3): 195-203. PubMed ID: 20331376 doi:10.3109/09593980903015250

11. Portney L, Watkins M. Foundations of Clinical Research: Application to Practice. Stamford, CT: Pearson Education Limited; 2013.

12. Weir JP. Quantifying test-retest reliability using the intraclass correlation coefficient and the sem. J Strength Cond Res. 2005; 19(1):231-240. PubMed ID: 15705040
13. Norkin CC, White DJ. Measurement of Joint Motion: A Guide to Goniometry. Philadelphia, PA: FA Davis Company; 2016.

14. Hibberd EE, Laudner K, Berkoff DJ, Kucera KL, Yu B, Myers JB. Comparison of upper extremity physical characteristics between adolescent competitive swimmers and nonoverhead athletes. $J$ Athl Train. 2016;51(1):65-69. doi:10.4085/1062-6050-51.2.04

15. Beach ML, Whitney SL, Dickoff-Hoffman S. Relationship of shoulder flexibility, strength, and endurance to shoulder pain in competitive swimmers. J Orthop Sports Phys Ther. 1992;16(6): 262-268. PubMed ID: 18796745 doi:10.2519/jospt.1992.16. 6.262 